\title{
IL SUICIDIO DI CESARE PAVESE (1908-1950)
}

\section{Giovanna Armellin Secchi}

\section{RESUMEN}

\begin{abstract}
César Pavese (1908-50) es un poeta y novelista italiano que ha traducido los escritos de varios americanos al italiano y ha escrito crítica literaria. Sus escritos antifascistas lo llevaron al encarcelamiento, lo que motivó en él la escritura creativa. Durante la Segunda Guerra Mundial, formó parte de la resistencia. Pavese fue encontrado muerto en la habitación de un hotel en Torino.

Por lo general, la ficción de Pavese versa sobre los conflictos de la vida contemporánea, entre ellos la búsqueda de una identidad propia. Esta búsqueda se da por ejemplo en $\mathrm{La}$ luna e i falò (1950) considerada su mejor novela.
\end{abstract}

\begin{abstract}
Cesare Pavese(1908-50), Italian poet and novelist. He also translated the writings of numerous Americans into Italian and wrote literary criticism. His anti-Fascist writings led to his imprisonment, which in turn led to his creative writing. During World War II he was part of the Resistence. Pavese's fiction generally deals with the conflicts of contemporary life, such as the search for self identity-as in The Moon and the Bonfires (1950), regarded as his best novel. He was found life less in his room in a hotel of Torino.
\end{abstract}

Lo scrittore italiano Cesare Pavese, importante rappresentante del neorealismo italiano, nasce nel 1908 a Santo Stefano Belbo nelle colline delle Langhe, in provincia di Cuneo, da una famiglia piccolo borghese di origine contadina. Ben presto rimane orfano di padre e si trasferisce a Torino, dove si suicida nel 1950.

Da questa breve sintesi biografica risaltano due fatti importanti: la prematura perdita del padre e l'allontanamento dalla campagna, due traumi che tormentano e accompagnano costantemente lo scrittore durante la sua corta vita.

A Torino, Pavese compie i suoi studi liceali e universitari e viene a contatto con amici impegnati nella lotta antifascista, come Leone Ginzburg. Si laurea in letteratura inglese e approfondisce soprattutto gli autori americani in auge in quel periodo.

La letteratura americana, infatti, considerata una cultura giovane in contrapposizione alla vecchia cultura europea, entra in Italia clandestinamente durante il periodo fascista, attraverso le opere di Pavese e di Vittorini, e Pavese cerca e ricava dagli scrittori americani una lezione di stile letterario. 
Cesare Pavese è considerato, con Elio Vittorini, fondatore del neorealismo, ma a differenza di quasi tutti gli esponenti del neorealismo, tipicamente aletterati, Pavese è molto colto.

L'immediato dopoguerra soffre nel campo letterario, come in quello sociale e politico, il disorientamento proprio dei periodi di transizione. Pavese è ritenuto dalla critica uno dei più tipici esponenti di questo disorientamento spirituale e della ricerca di una comunicazione piú autentica.

Sviluppa di preferenza il tema della solitudine inquieta, perpetuamente ripiegata su se stessa in un'analisi che trova la sua forma congeniale nella rievocazione retrospettiva e nel diario intimistico. Uno sfogo che si insabbia in un disperato cerebralismo chiuso ad ogni reale sforzo di comunicazione. Natalia Ginzburg scrive di Pavese, dopo la sua morte, in Ritratto d'un amico:

\footnotetext{
Era, qualche volta, cosí triste, e noi avremmo pur voluto venirgli in aiuto: ma non ci permise mai una parola pietosa, un cenno di consolazione: e accadde anzi che noi, imitando i suoi modi, respingessimo nell'ora del nostro sconforto la sua misericordia. Non fu, per noi, un maestro, pur avendoci insegnato tante cose: perché vedevamo bene le assurde e tortuose complicazioni di pensiero, nelle quali imprigionava la sua semplice anima; e avremmo anche noi voluto insegnargli qualcosa, insegnargli a vivere in un modo piú elementare e respirabile: ma non ci riuscì mai d'insegnargli nulla, perché quando tentavamo di esporgli le nostre ragioni, alzava una mano e diceva che lui sapeva già tutto (Ginzburg 1976: 30).
}

Le componenti letterarie e filosofiche del periodo neorealistico si rifanno al contesto socio-culturale contemporaneo. L'esistenzialismo che pone l'accento sulla concreta esistenza umana, ed in particolare l'esistenzialismo ateo di Sartre e di Camus, si cala nel grigiore quotidiano comunicandogli il senso del disagio, della solitudine e obbligando l'uomo ad accettare il proprio limite terreno e assurdo secondo la linea esistenziale in uno squallore di incomunicabilità e di non amore. Il dopoguerra, del resto, è il momento più favorevole per l'affermarsi di un simile clima.

Analizzando il mondo che ci circonda, si può notare quanto pessimismo infirma la vita e il tragico della situazione dell'uomo moderno, e il motivo fondamentale del suo complesso di solitudine sta proprio nel fatto di non provare nessuna comunicazione spirituale, di non avere interlocutori, e non si possono negare, dunque, il fatto e gli effetti disastrosi di questa strordinaria solitudine. Chiunque si sia sollevato al di sopra della banalità quotidiana, come Pavese, non può far altro che sentirsi solo e abbandonato. L'uomo Pavese è infelice perché incapace di realizzare la comunione con gli altri, comunione che è il presupposto per essere felice. La filosofia della solitudine e della conseguente infelicità si addice allo scrittore, che è per natura predisposto al pessimismo. Di lui, la scrittrice e amica Natalia Ginzburg scrive:

\footnotetext{
Ma ci pensava in un modo che si faceva, con gli anni, sempre piú complicato e tortuoso, che non ne poteva germogliare nessuna semplice conclusione. Si era creato, con gli anni, un sistema di pensieri e di principi cosí aggrovigliato e inesorabile, da vietargli l'attuazione della realtà piú semplice: e quanto piú proibita e impossibile si faceva quella semplice realtà, tanto piú profondo in lui diventava il desiderio di conquistarla, aggrovigliandosi e ramificando come una vegetazione tortuosa e soffocante (Ginzburg 1976: 30).
}

L'incomunicabilità dei personaggi dei suoi romanzi, il senso di vuoto interiore e di solitudine angosciata è la logica conseguenza del venir meno di ogni valore universale, infatti, privo di una base di principi sui quali potersi intendere con comune giudizio, si chiude in una 
disperata, invalicabile solitudine che si esprime nel soliloquio dell'angoscia, nell'insistito monologare. Confessa Pavese:

Tutto il dramma della vita è sempre questo: come rompere il dramma della propria solitudine e come comunicare con gli altri (Pazzaglia 1972: 1181).

Il neorealismo, di cui lo scrittore è tra i massimi rappresentanti, partito da un positivo bisogno di proporre come centro d'interesse della nuova letteratura il popolo nella sua concretezza esistenziale, col suo concreto disagio spirituale e sociale, non sa poi uscire dal vicolo desolato e arido della miseria, intesa nella sua forma più vasta, per additare una soluzione costruttiva.

Pavese è testimone di questo stato di incertezza e di lacerazione interiore da cui non ha la forza di uscire, e paga con la rinuncia volontaria ad una vita che gli si rivela in tutta la sua esasperante povertà.

Ma quali sono le cause del tragico fallimento di uno sforzo di avvicinarsi all'uomo, per condividere con fraterna partecipazione la sua tragedia, attraverso la denuncia di una crisi sociale, per aiutarlo a liberarsi dal peso dell'angoscia?

L'uomo Pavese non trova alcun ancoraggio sicuro a cui poter affidare una residua aspirazione di bontà già scossa dall'esperienza della guerra, un senso di stanchezza e di delusione, perché rileva che il richiamo ad una fratellanza umana manca di un'intrinseca forza interiore, incapace di penetrare e di risolvere i problemi.

Per capire il mondo interiore di Pavese, è necessario esaminare l'ultimo romanzo La luna e i falò considerato il suo capolavoro, che è pubblicato nel 1950, l'anno della sua morte. Qui troviamo la sua arte e i suoi drammi con sfondi decadentistici e crepuscolari. Il mito, la nostalgia per l'infanzia passata e irrecuperabile, irreversibile, l'amore per le colline e per i contadini, il giudizio negativo per i borghesi e per il clericalismo. Tutto il romanzo è intriso di nostalgia per il passato irreversibile, che neanche con la memoria si può recuperare, per la fine dei miti dell'infanzia e delle speranze distrutte dalle esperienze della maturità.

L'influenza del decadentismo induce Pavese al fascino della solitudine, dell'angoscia, della morte e del suicidio. L'idea del suicidio lo accompagna da molto tempo, in un suo racconto scritto nel '39 Suicidi dice:

Dovrei piangere anch'io, Carlotta. Ma so che non serve. Tutto questo che tu provi, l'ho provato. Sono stato per uccidermi e poi mi è mancato il coraggio. Questa è la burla: chi è tanto debole da pensare al suicidio è troppo debole per farlo (Pavese 1968: 304).

Però, a differenza degli scrittori decadenti, che si abbandonano al mistero e al sentimentalismo, Pavese cerca disperatamente una soluzione, crede di averla trovata nel mito, si sforza tutta la vita per chiarire i suoi miti, ma l'inutile tentativo lo porta al suicidio. Proprio nel 1950, quando esce il suo romanzo La luna e i falò, e riceve il Premio Strega, poco dopo si suicida in una camera d'albergo di Torino, logica conseguenza della sua parabola umana e letteraria.

Come Jean-Paul Sartre, che nel '64 non accetta il Premio Nobel assegnatogli, stupisce l'opinione pubblica; ma in fondo, quel gesto clamoroso non è che un'espressione di concreta coerenza ai suoi principi; sottolinea il disprezzo verso una società che non approva. 
Pavese cerca una soluzione alla difficile esistenza sua e degli altri, però, neppure l'impegno politico, come antifascista, partigiano e comunista lo riscatta da quell' angoscia che gli è propria, anzi la perdita delle illusioni nate con la resistenza, la accentua in forma abnorme.

Ti ricordi i discorsi che facevamo con tuo padre nella bottega? Lui diceva già allora che gli ignoranti saranno sempre ignoranti, perché la forza è nelle mani di chi ha interesse che la gente non capisca, nelle mani del governo, dei neri, dei capitalisti...Qui alla Mora era niente, ma quand'ho fatto il soldato e girato i carrugi e i cantieri a Genova ho capito cosa sono i padroni, i capitalisti, i militari...Allora c'erano i fascisti e queste cose non si potevano dire... Ma c'erano anche gli altri... (Pavese 1982: 104).

L'autore di La luna e $i$ falò insegue una effettiva e costruttiva comunicazione con gli uomini, si impegna nella creazione di una società nuova e autentica. Ma nonostante l'impegno politico, dovuto alla indole vive e sente, maggiormente il suo dramma personale più di quello sociale.

Gli diceva che sono soltanto i cani che abbaiano e saltano addosso ai cani forestieri e che il padrone aizza un cane per interesse, per restare padrone, ma se i cani non fossero bestie si metterebbero d'accordo e abbaierebbero addosso al padrone. (...) ch'era come la guerra che s'era fatta nel '18 - tanti cani scatenati dal padrone perché si ammazzassero e i padroni restare a comandare. Diceva che basta leggere il giornale -i giornali di allora- per capire che il mondo è pieno di padroni che aizzano $\mathrm{i}$ cani (Pavese 1982: 75-76).

Non resta per Pavese che l'arte come unica risorsa per trovare una soluzione al problema esistenziale, per la letteratura si sacrifica tutta la vita, crede di poter trovare nella poesia una giustificazione al vuoto dell'esistenza, una ragione per l'esistenza:

Per Pavese la letteratura è al primo posto come valore assoluto esistenziale, tanto che è la letteratura a dare senso alla vita, ad arricchirla di valori e di significati, la letteratura è il centro e sulla letteratura si concentrano tutte le ansie, tutte le più forti suggestioni esistenziali di Pavese, fino al punto da creare una tensione insostenibile che mette in crisi la sua stessa esistenza (Ricciardi 1992: 943).

La tragedia dell'uomo Pavese consiste nel desiderio insoddisfatto di trovare colloquio e amore tra i suoi simili. Questa sua frustrata ricerca lo porta a chiudersi sempre più nella propria solitudine. Il fondo del problema sta nell'incomunicabilità con gli uomini e con Dio.

Quest'estate sono sceso all'albergo dell'Angelo, sulla piazza del paese, dove piú nessuno mi conosceva, tanto sono grand'e grosso. Neanch'io in paese conoscevo nessuno; ai miei tempi ci si veniva di rado, si viveva sulla strada, per le rive, nelle aie.(...) Stessi rumori, stesso vino, stesse facce di una volta. (...) E le allegrie, le tragedie, le promesse in riva al Belbo. C'era di nuovo che una volta(...).C'era di nuovo che adesso lo sapevo, e quel tempo era passato (Pavese 1982: 11).

Incapace di uscire da quel vuoto esistenziale con l'impegno sociale o con l'amore, da quel senso profondo d'insoddisfazione che va parallelo all'incomunicabilità con gli uomini e con Dio, arriva al suicidio.

Anche nell'amore cerca una ragione d'esistenza, ma tutte le sue esperienze amorose sono minate dall'impossibilità di abbandonarsi, di comunicarsi, di stringere un legame, questa sua incapacità di comunicabilità, quella ricerca costante di ciò che non può raggiungere, lo portano al fallimento delle sue relazioni amorose: 
Quando conobbi Carlotta, uscivo da una burrasca che per poco non m'era costata la vita; e provavo un'amara ilarità a ritornare per le vie deserte fuggendo da chi mi amava. Per tanto tempo era toccato a me di passare le notti e i giorni umiliato e inferocito dal capriccio di una donna (Pavese 1968: 300).

Pavese infine confessa e riconosce che la causa del fallimento si deve attribuire sempre e solo a noi stessi:

Ora sono convinto che nessuna passione ha tanta forza da mutare la natura di chi la subisce. Si può morirci, ma le cose non cambiano. (...) O meglio: si è veduta nella crisi la propria vera natura, e questa c'inorridisce e la normalità ci disgusta, e si vorrebbe magari essere morti tanto l'insulto che ci è fatto è atroce, ma non c'è altri da accusare se non noi (Pavese 1968: 300).

Neppure in Dio può trovare conforto e appoggio perché non ci crede, lo scrittore è iscritto al partito comunista che è per la chiesa considerato sovversivo, da piccolo invece ci credeva, ed ecco ancora una volta il contrasto tra infanzia e maturità che risalta da questo brano dove dei sacerdoti scrive:

Così sotto quel sole, sugli scalini della chiesa, da quanto tempo non sentivo piú la voce di un prete dir la sua. E pensare che da ragazzo quando la Virgilia ci portava a messa, credevo che la voce del prete fosse qualcosa come il tuono, come il cielo, come le stagioni che servissero alle campagne, ai raccolti, alla salute dei vivi e dei morti. Adesso mi accorsi che i morti servivano a lui. Non bisogna invecchiare né conoscere il mondo. (...) Coi morti i preti hanno sempre ragione (Pavese 1982: 52).

Con La luna e i falò lo scrittore s'immerge con la memoria nel mondo dell'infanzia e la realtà attuale e i ricordi si intrecciano, il presente e il passato si intersecano liricamente, ma il contrasto delle due realtà provoca una tensione insostenibile per lo scrittore che scrive:

\begin{abstract}
-Sì, sì giovanotti, sì, sì ragazze... pensate a crescere... così dicevano i nostri nonni ... si vedrà quando toccherà a voi. A quei tempi non mi capacitavo che cosa fosse questo crescere, credevo fosse solamente fare delle cose difficili, come comprare una coppia di buoi, fare il prezzo dell'uva, manovrare la trebbiatrice. Non sapevo che crescere vuol dire andarsene, invecchiare, veder morire, ritrovare la Mora com'era adesso (Pavese 1982: 57-58).
\end{abstract}

I contrasti tra la città e la campagna, tra i contadini e i borghesi, e soprattutto tra l'infanzia e la maturità sono i temi familiari e prediletti dallo scrittore. I temi del ritorno, del paese natale, delle colline, della campagna, dell'infanzia i cui ricordi restano sfocati dal passaggio del tempo, la memoria, a Pavese sembra di trovare nei ricordi un appoggio, ma si rende poi conto che neppure questi possono essere una ragione di vita.

All'origine della poetica pavesiana c'è la scoperta dell'infanzia come l'età in cui l'uomo compie le sue
esperienze fondamentali. E nell'infanzia che si ha il primo contatto con il mondo e che si creano i simboli,
i miti, corrispondenti alle singole rivelazioni delle cose, che poi vivranno nella coscienza come forme
dell'immaginazione trascendentale. Le successive esperienze -il maturare dell'uomo nel corso degli anni
non sono che un conoscere una seconda volta, un riscoprire e ridurre a chiarezza quei miti (Salinari 1976:
1225).

Nel suo ultimo romanzo, La luna e $i$ falò i temi fondamentali sono la solitudine dell'uomo, dell'esule che sente nostalgia della sua gente e dei suoi luoghi e che trova al ritorno, con delusione, la stessa solitudine: 
Quella notte, prima di scendere a Oakland (...), capii nel buio, in quell'odore di giardino e di pini, che quelle stelle non erano le mie, che come Nora e gli avventori mi facevano paura. Le uova al lardo, le buone paghe, le arance grosse come angurie, non erano niente, somigliavano a quei grilli e a quei rospi. Valeva la pena esser venuto? Dove potevo andare? Buttarmi dal molo? (Pavese 1982: 17).

Tante domande, tanti interrogativi che non trovano una risposta adeguata per l'autore, troppo esigente, troppo colto per accettare quella vita vuota di valori assoluti. Le sue opere, quasi sempre a sfondo autobiografico, ci riportano nelle sue colline, nel suo paese, che ama e descrive con trasporto, con nostalgia e rimpianto, ma anche il suo paese lo delude, perchè non vi trova la comunicazione sperata. Infatti scrive:

Un paese ci vuole, non fosse che per il gusto di andarsene via. Un paese vuol dire non essere soli, sapere che nella gente, nelle piante, nella terra c'è qualcosa di tuo, che anche quando non ci sei resta ad aspettarti. Ma non è facile starci tranquillo. Da un anno che lo tengo d'occhio e quando posso ci scappo da Genova, mi sfugge di mano. Queste cose si capiscono col tempo e l'esperienza. Possibile che a quarant'anni, e con tutto il mondo che ho visto, non sappia ancora che cos'è il mio paese? (Pavese 1982: 9).

Il costante senso di solitudine, di incomunicabilità, d'insoddisfazione, di tormento e d'angoscia che pervade l'uomo e il letterato Pavese, non si può attribuire solo alle circostanze socio-politiche del momento, che certamente influiscono, ma che sono assorbite, dallo scrittore che è per indole pessimista, in modo sproporzionato. Pur diventato famoso, quando nel ' 50 riceve il premio Strega per la letteratura che significa per lui un valore assoluto, si suicida. Difatti per questo scrittore colto ed estremamente esigente, le cose raggiunte non lo soddisfano piú, è incapace di godere e di comunicare, perché è incapace di amare gli altri e se stesso. Per Pavese la vita è come un falò, bruciati tutti i miti, non resta che la cenere.

\section{Bibiografía}

Ascor Rosa, Alberto. 1969. Scrittori e popolo. Roma: Savelli.

Ginzburg, Natalia. 1976. Le piccole virtù. Torino: Einaudi.

Manacorda, Giuliano. 1972. Storia della letteratura italiana contemporanea. (1940-1965). Roma: Editori Riuniti.

Pavese, Cesare. 1941. Paesi tuoi. Torino: Einaudi.

1948. La casa in collina. Torino: Einaudi.

1949. Tra donne sole. Torino: Einaudi.

1949. Prima che il gallo canti. Torino: Einaudi.

1968. Opere. Torino: Einaudi. 
1968. Racconti. Torino: Einaudi.

1982. La luna e i falò. Torino: Einaudi.

Pullini, Giorgio. 1965. Il romanzo italiano del dopoguerra. Padova: Marsiglio.

Ricciardi, Mario. 1992. La letteratura in Italia. Milano: Bompiani.

Salinari, Carlo. 1976. Storia della letteratura italiana. Roma-Bari: Editori Laterza. 
n 